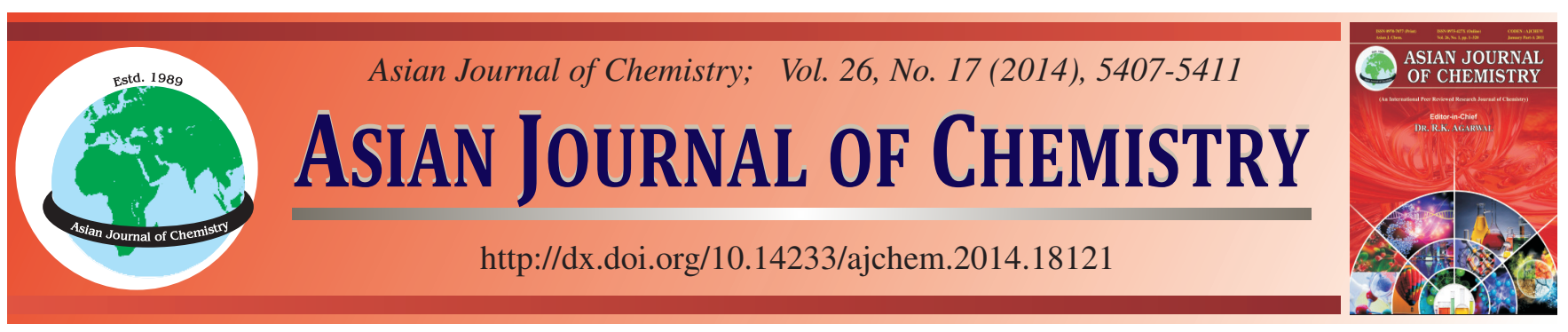

\title{
Spin-Glass Behaviour and Magnetic Properties of Multi-Metallic Prussian Blue Compound $\mathrm{Co}_{0.25} \mathrm{Mn}_{1.25}\left[\mathrm{Fe}(\mathrm{CN})_{6}\right] \cdot 5.9 \mathrm{H}_{2} \mathrm{O} \dagger$
}

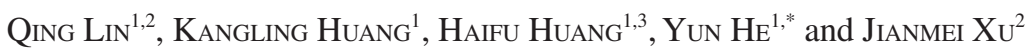

${ }^{1}$ College of Physics and Technology, Guangxi Normal University, Guilin 541004, P.R. China

${ }^{2}$ Department of Information Technology, Hainan Medical College, Haikou 571101, P.R. China

${ }^{3}$ Department of Physics, Nanjing University, Nanjing 210093, P.R. China

*Corresponding author: Fax: +86 773 5840785; Tel: +86 773 5811173; E-mail: hy@gxnu.edu.cn

Multi-metal prussian blue compound $\mathrm{Co}_{0.25} \mathrm{Mn}_{1.25}\left[\mathrm{Fe}(\mathrm{CN})_{6}\right] \cdot 5.9 \mathrm{H}_{2} \mathrm{O}$ has been synthesized.The temperature-dependent magnetic susceptibilities indicate that there exist a ferrimagnetic interaction in the compound and magnetic transition at $9.5 \mathrm{~K}$. The Curie constant of $\mathrm{C}=6.59 \mathrm{~cm}^{3} \mathrm{~K} \mathrm{~mol}^{-1}$ and Curie temperature of $\theta=-11.28 \mathrm{~K}$ were observed in fitting according to Curie-Weiss law. The observed value of coercive field and remanent magnetization at $4 \mathrm{~K}$ for the compound are $230 \mathrm{Oe}$ and $0.072 \mu_{\beta}$. The presence of spin-glass behaviours in the compound is ascribed mainly to domain mobility or domain growth under different cooling conditions. The behaviour of $\chi^{\prime}$ and $\chi^{\prime \prime}$ is typical of a spin glass statego through a maximum with strong frequency dependence. The observed values for the isomer shift, the quadrupole splitting is $0.41(9) \mathrm{mm} / \mathrm{s}$, I.S. is $-0.15(4) \mathrm{mm} / \mathrm{s}$, values which are characteristic of low-spin $\mathrm{Fe}(\mathrm{III})(\mathrm{S}=1 / 2)$ ions. The Mössbauer spectroscopy study revealed the presence of low spin Fe(III) $(\mathrm{S}=1 / 2)$.

Keywords: Prussian blue analogue, Magnetic, Spin glass, Magetic transition, Mössbauer spectroscopy.

\section{INTRODUCTION}

Compared with previous ion or alloy magnet, molecularbased magnetic material is studied, designed and prepared on the bases of the molecular level and may be obtained through the solution method because of its synthetic conditions of mild $^{1,2}$. At the same time, it is likely to bring a breakthrough in the field of magnetic materials, due to the infinity of molecular synthesis and the diversity of selection for the ions and ligand. But for most molecular-based magnet, their magnetic transition temperature $\left(\mathrm{T}_{\mathrm{C}}\right)$ is lower than room temperature so that practical application is limited. Therefore design and preparation of molecular-based magnetic material with a special magnetic properties which can be controlled, particularly with high Curie temperature $T_{c}$, are one of the main challenges in the study of molecular-based ferromagnet. Hashimoto et al. have prepared a series of molecular alloy magnet $\left[\mathrm{A}^{\mathrm{II}}{ }_{\mathrm{x}} \mathrm{B}^{\mathrm{II}}{ }_{1-\mathrm{x}}\right]_{\mathrm{y}}\left[\mathrm{Cr}(\mathrm{III})(\mathrm{CN})_{6}\right] \cdot \mathrm{nH}_{2} \mathrm{O}$, which magnetic parameters like saturation magnetization $\left(\mathrm{M}_{\mathrm{s}}\right)$, Weiss paramagnetic Curie temperature $(\theta)$, magnetization $(\mathrm{M})$, coercive field $\left(\mathrm{H}_{\mathrm{c}}\right)$, transition temperature $\left(\mathrm{T}_{\mathrm{c}}\right)$, compensation temperature $\left(\mathrm{T}_{\text {comp }}\right)$, etc. ${ }^{3-5}$ can be controlled through changing the value of $\mathrm{x}$ or metal cations. Previous workers have synthe- sized a number of prussian blue analogues $\mathrm{C}_{\mathrm{n}} \mathrm{A}_{\mathrm{P}}\left[\mathrm{B}(\mathrm{CN})_{6}\right]_{\mathrm{q}} \cdot \mathrm{xH}_{2} \mathrm{O}$ (Molecular structure of prussian blue analogue compound as shown in Fig. 1). Among these molecular alloy magnet, it exhibits some special magnetic phenomenon, such like the spontaneous magnetization changes its sign twice with changing temperature, one or even two compensation temperatures, photoinduced magnetic-pole inversion, an inverted hysteresis loop, etc. So, the ideas of synthesis for the type of moleculebased magnets can provide a new way to overcome some difficulties on the study of molecular-based magnet. In the paper, we have synthesized multi-metal Prussisan blue compound $\mathrm{Co}_{0.25} \mathrm{Mn}_{1.25}\left[\mathrm{Fe}(\mathrm{CN})_{6}\right] \cdot 5.9 \mathrm{H}_{2} \mathrm{O}$ by co-precipitation method and have studied the magnetic properties of this compound.

\section{EXPERIMENTAL}

$\mathrm{CoCl}_{2} \cdot 6 \mathrm{H}_{2} \mathrm{O}, \mathrm{Mn}\left(\mathrm{SO}_{4}\right)_{2} \cdot 6 \mathrm{H}_{2} \mathrm{O}$ and $\mathrm{K}_{3} \mathrm{Fe}(\mathrm{CN})_{6}$ are reagent grade and without further purification. Elemental analysis (C, N, H) were performed on Perk-Elmer2400 II analyser. IR spectrum were recorded on a Perkin-Elmer FT-IR spectrophotometer as $\mathrm{KBr}$ pellet in the $4000-400 \mathrm{~cm}^{-1}$ range. Magnetization measurements were measured on a Quantum Design MPMS-5 magnetometer in the scope of 2-300 K. 


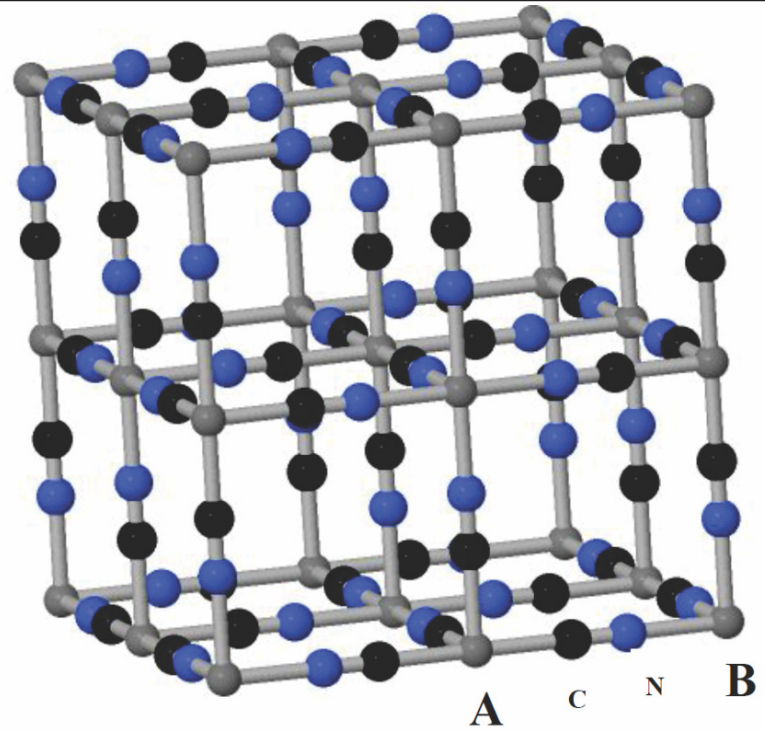

Fig. 1. Structure of Prussian blue compound

Synthesis of $\mathrm{Co}_{0.25} \mathrm{Mn}_{1.25}\left[\mathrm{Fe}(\mathrm{CN})_{6}\right] \cdot 5.9 \mathrm{H}_{2} \mathrm{O}$ : Polycrystaline compound was prepared by coprecipitation method. To a solution of $25 \mathrm{~mL} \mathrm{CoCl} 2 \cdot 6 \mathrm{H}_{2} \mathrm{O}(0.25 \mathrm{mmol})$ was mixed 125 $\mathrm{mL} \mathrm{Mn}\left(\mathrm{SO}_{4}\right)_{2} \cdot 6 \mathrm{H}_{2} \mathrm{O}(1.25 \mathrm{mmol})$ solution. Then a solution of $100 \mathrm{~mL} \mathrm{~K}_{3} \mathrm{Fe}(\mathrm{CN})_{6}(1 \mathrm{mmol})$ was slowly added to the mixed solution of $\mathrm{CoCl}_{2} \cdot 6 \mathrm{H}_{2} \mathrm{O}$ and $\mathrm{Mn}\left(\mathrm{SO}_{4}\right)_{2} \cdot 6 \mathrm{H}_{2} \mathrm{O}$ and a solid was precipitated immediately. After $48 \mathrm{~h}$, the precipitates were filtered, washed repeatedly with water and dried at $45{ }^{\circ} \mathrm{C}$. Elemental analysis to measure $\mathrm{C}, \mathrm{N}, \mathrm{H}$ mass ratio: found: $\mathrm{C}$, 17.44; N, $21.35 \%$; H, 3.06; calcd: C, 17.94; N, $20.92 \%$; H, 2.96.

IR spectrum: The IR spectrum of compound has been recorded over the 4000-400 $\mathrm{cm}^{-1}$ range and shown in Fig. 2. It shows two bands at 2078 and $2151 \mathrm{~cm}^{-1}$ indicating the existence of two types of cyanide groups in the crystal lattice of compound $^{6,7}$. Compounds are easily identified by their stretching frequencies in 2200-2000 $\mathrm{cm}^{-1}$ range, which are consistent with the formation of bridging cyanide groups and there are two different coordination environment. The broad peak at 3404 and $1611.5 \mathrm{~cm}^{-1}$ are assigned to the $\mathrm{v}(\mathrm{O}-\mathrm{H})$ of the crystal water stretching vibrations.

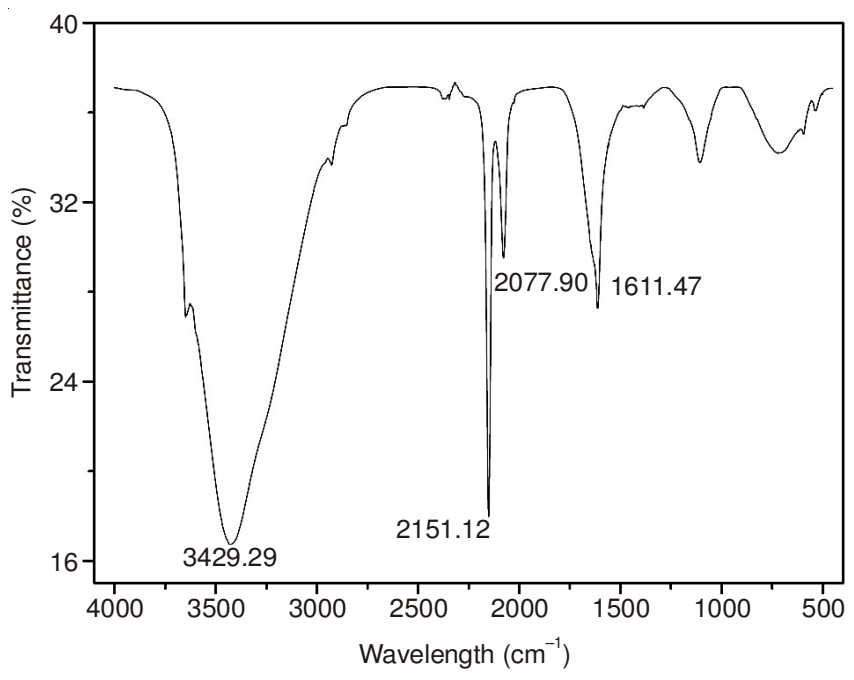

Fig. 2. FT-IR spectrum of the Prussian blue

\section{RESULTS AND DISCUSSION}

DC magnetic susceptibility: The magnetic susceptibility of the compound $\mathrm{Co}_{0.25} \mathrm{Mn}_{1.25}\left[\mathrm{Fe}(\mathrm{CN})_{6}\right] \cdot 5.9 \mathrm{H}_{2} \mathrm{O}$ was measured from 2-300 K (Fig. 3), which corresponds to the steepest rise of magnetization with decreasing temperature. A magetic transition temperature $\left(\mathrm{T}_{\mathrm{c}}\right)$ of $9.5 \mathrm{~K}$ is estimated by minima of $\mathrm{dM} / \mathrm{dT}$ vs T curve. According to $\chi_{\mathrm{m}}{ }^{-1}$ versus $\mathrm{T}$ curve (Fig. 4), in $300-20 \mathrm{~K}$ range, the values of $\chi_{\mathrm{m}}$ gradually increase and then sharply increase after $20 \mathrm{~K}$ with a further decrease of the temperature. The curves of $\chi_{\mathrm{m}}{ }^{-1} v s \mathrm{~T}$ above $30 \mathrm{~K}$ obey the CurieWeiss law with a Curie constant of $\mathrm{C}=6.59 \mathrm{~cm}^{3} \mathrm{~K} \mathrm{~mol}^{-1}$ and Weiss paramagnetic Curie temperature of $\theta=-11.28 \mathrm{~K}$. The values of $\mathrm{T}_{c}, \theta$ and $\mathrm{C}$ in $0.5 \mathrm{KOe}$ are different from those values for ferrimagnet $\mathrm{Co}_{1.5}\left[\mathrm{Fe}(\mathrm{CN})_{6}\right] \cdot 6 \mathrm{H}_{2} \mathrm{O}\left(\mathrm{T}_{\mathrm{c}}=16 \mathrm{~K}, \mathrm{C}=5.4 \mathrm{~cm}^{3}\right.$ $\left.\mathrm{K} \mathrm{mol}^{-1}, \theta=-15 \mathrm{~K}\right)^{8}$.

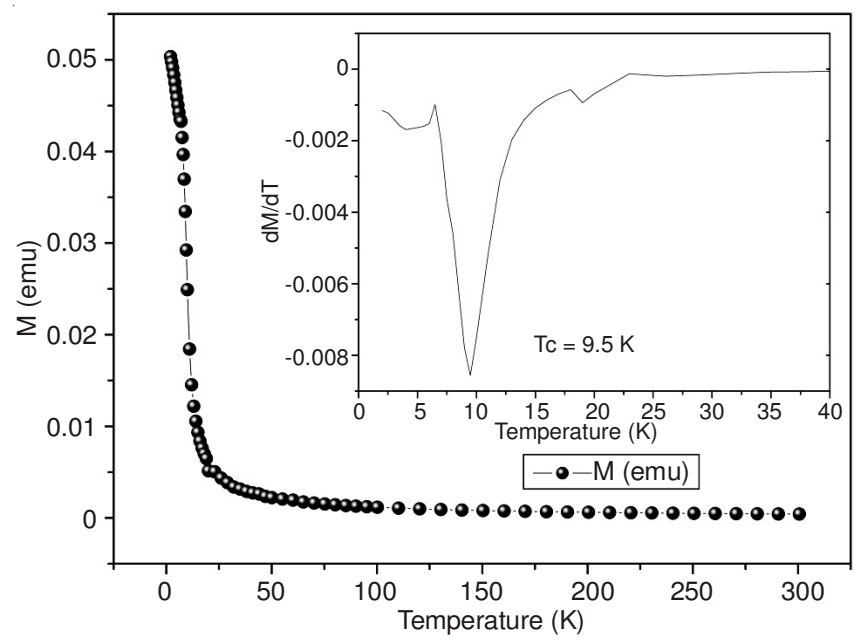

Fig. 3. M vs. T for the compound (Inset: plot of $\mathrm{dM} / \mathrm{dT} v s . \mathrm{T}$ )

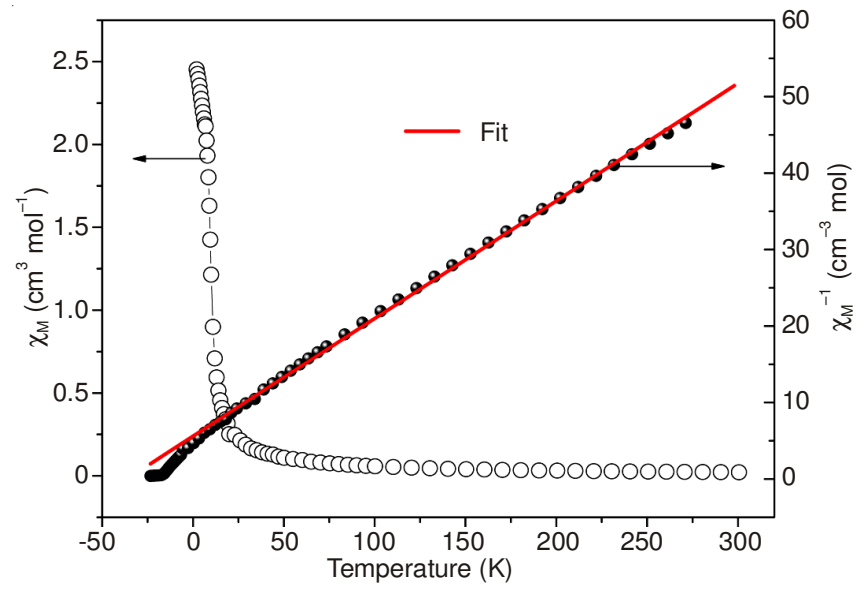

Fig. 4. $\chi_{\mathrm{m}}$ and $\chi_{\mathrm{m}}{ }^{-1} v s$. $\mathrm{T}$ for the compound

A curve of $\chi_{\mathrm{m}} \mathrm{T} v s . \mathrm{T}$ is shown in Fig. 5. The effective moment $\chi_{\mathrm{m}} \mathrm{T}$ sharply increase to reach maximum of $15.61 \mathrm{~cm}^{3}$ $\mathrm{K} \mathrm{mol}^{-1}$ at $8 \mathrm{~K}$ and final decrease at lower temperature, indicating antiferromagnetic interaction ${ }^{8,9}$. A curve of $\mu_{\text {eff }} v s$. $\mathrm{T}$ is shown in Fig. 6 . The effective moment $\mu_{\text {eff }}$ first slowly decrease to reach a minima of $6.48 \mu_{\beta}$ at $31 \mathrm{~K}$, then sharply increase to reach maximum of $11.11 \mu_{\beta}$ at $8 \mathrm{~K}$ and final decrease at lower temperature, indicating antiferromagnetic interaction between paramagnetic centers. This kind of behaviour is a characteristic of a ferrimagnet ${ }^{8,9}$. 


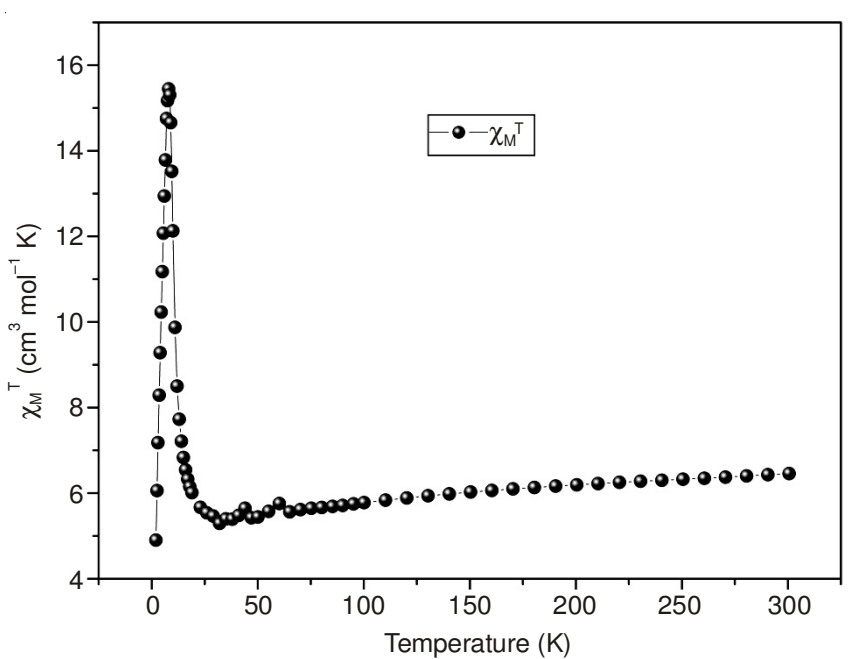

Fig. 5. $\chi_{\mathrm{m}} \mathrm{T}$ versus $\mathrm{T}$ plot of the compound

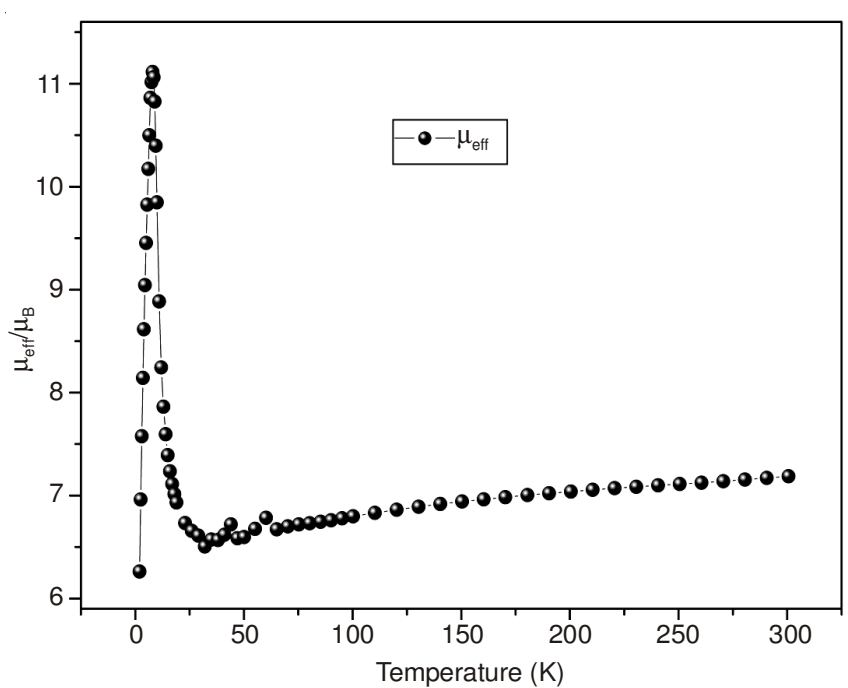

Fig. 6. $\mu_{\mathrm{eff}}$ versus $\mathrm{T}$ plot of for the compound

Zero-field-cooled and field-cooled magnetization:

Fig. 7 shows the curves of Zero-field-cooled and field-cooled magnetization of compound at different field $\mathrm{H}=20,150$, 250, 500 Oe. The values of $M$ increase and exhibit weak irrevers-ibility in the field-cooled (FC) magnetization curves below $\mathrm{T}_{\mathrm{c}}=9.5 \mathrm{~K}$. There are a clear bifurcation phenomenon in the compound $\mathrm{Co}_{0.25} \mathrm{Mn}_{1.25}\left[\mathrm{Fe}(\mathrm{CN})_{6}\right] \cdot 5.9 \mathrm{H}_{2} \mathrm{O}$ by the fieldcooled $\left(\mathrm{M}_{\mathrm{FC}}\right)$ and zero-field-cooled $\left(\mathrm{M}_{\mathrm{ZFC}}\right)$ magnetization curves. $T_{\text {irr }}$ is a bifurcation temperature point of which fieldcooled and zero field-cooled magnetization curves separates out. In addition, the behaviour that $\mathrm{M}_{\mathrm{ZFC}}$ exhibits a maximum below $\mathrm{T}_{\text {irr }}$ is attributed to the cooperative freezing of spin glass. The irreversible behaviour of $\mathrm{M}$ and shift of bifurcation point to lower temperature with increasing $\mathrm{H}$ are characteristic for spin glasses ${ }^{10,11}$.

AC magnetic susceptibility: It is also confirmed that there exist a spin-glass behaviour in the compound $\mathrm{Co}_{0.25} \mathrm{Mn}_{1.25}\left[\mathrm{Fe}(\mathrm{CN})_{6}\right] \cdot 5.9 \mathrm{H}_{2} \mathrm{O}$ through $\mathrm{AC}$ magnetization curves. The AC magnetic susceptibility of the compound was measured at 4 Oe AC amplitude with zero-applied DC field in the frequencies 10-1000 $\mathrm{Hz}$ range, as shown in Fig. 8. It can be observed that in-phase (real) susceptibility, $\chi^{\prime}(\mathrm{T})$ curves

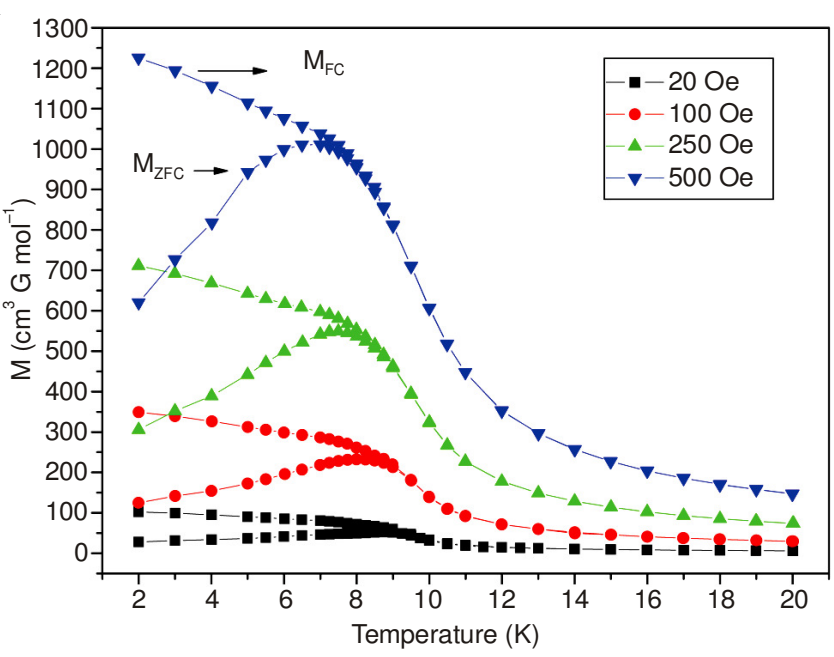

Fig. 7. ZFC and FC magnetization curves with different field
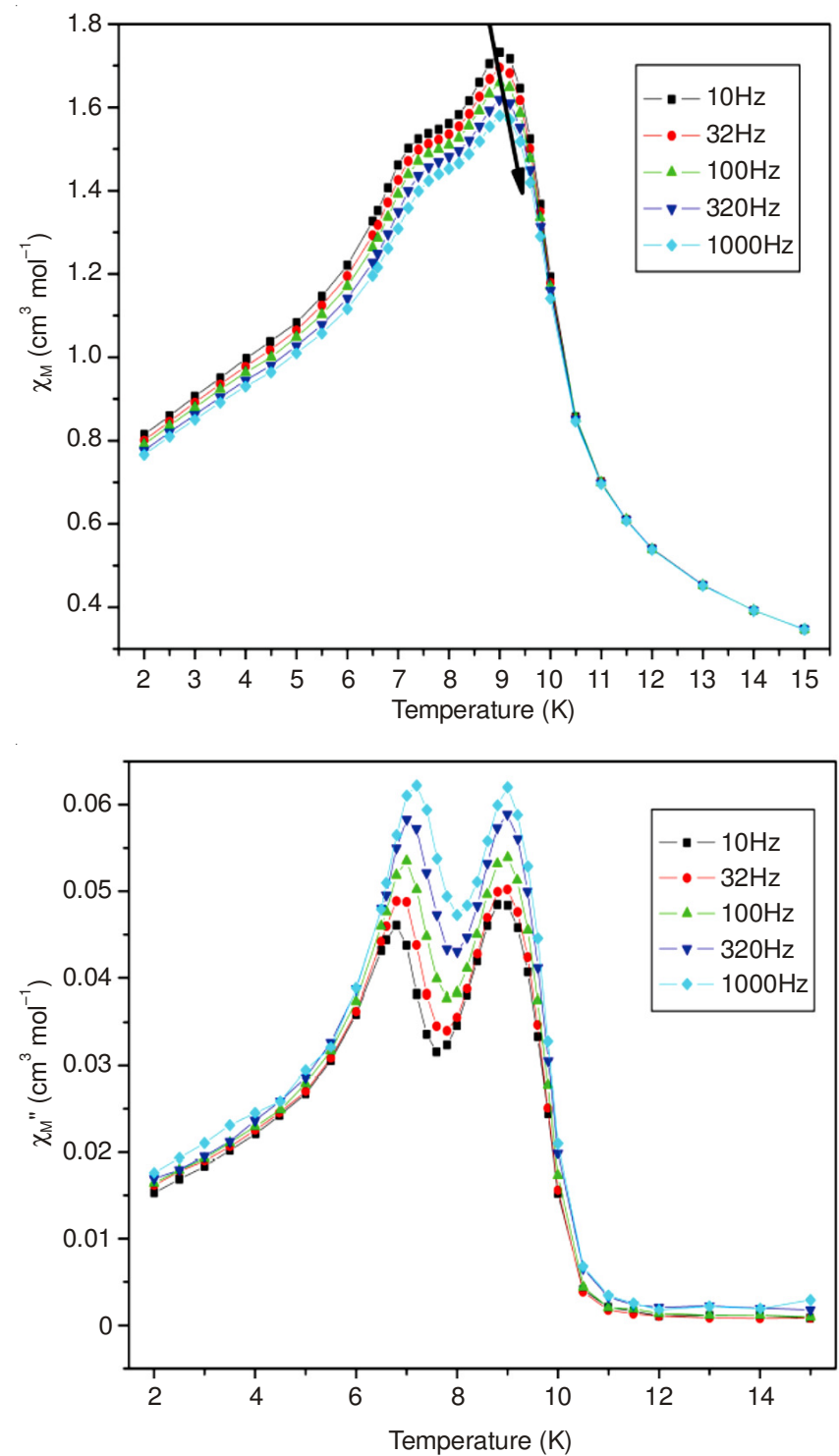

Fig. 8. $\quad \chi^{\prime}(\mathrm{T})$ and $\chi^{\prime \prime}(\mathrm{T})$ curves of AC magnetic susceptibility of the sample with different frequencies

exhibited a peak at about $9 \mathrm{~K}$ and the peak temperature $\mathrm{T}_{\mathrm{f}}(\mathrm{w})$ shifts toward higher temperatures with increasing frequency. That the $\chi(\mathrm{T})$ clearly shows a frequency dependence is typically 
assigned to spin glasses ${ }^{12-14}$. The relative shift of frequency (c) is $\mathrm{c}=\frac{\Delta \mathrm{T}_{\mathrm{f}}}{\mathrm{T}_{\mathrm{f}} \Delta \log \mathrm{w}}=0.006$, which fall within the range typical $\left(10^{-2}-10^{-3}\right)$ for the conventional spin-glass system. In addition, the $\chi$ ' show a broad peak at $7 \mathrm{~K}$, but out-of-phase (imaginary) susceptibility ${ }^{14-16}$ ], $\chi$ " (T) show two clear peak at 7 and $9 \mathrm{~K}$, as shown in Fig. 8, respectively and the peak temperature shifts toward higher temperatures with increasing frequency.

Field-dependent of magnetization and hysteresis behaviour: The ferrimagnetic behaviour is further characterized by the measurements of hysteresis behaviour and field-dependent magnetization (Fig. 9). Field-dependent magnetization curves measured at $4 \mathrm{~K}$ shows that a rapid increase in $\mathrm{M}$ at low magnetic field and then the values of $\mathrm{M}$ gradually increase with a further increase of the magnetic field ${ }^{17-20}$. The observed $\mathrm{M}_{\mathrm{s}}$ value is $4.31 \mu_{\beta}$, at $50 \mathrm{kOe}$, but the compound does not reach full saturation. The hysteresis curves is measured at $4 \mathrm{~K}$, shown in the Fig. 10. The coercive field $\left(\mathrm{H}_{\mathrm{c}}\right)$ value of $230 \mathrm{Oe}$ and remanent magnetization $\left(\mathrm{M}_{\mathrm{r}}\right)$ value of $0.072 \mu_{\beta}$ for the compound are smaller than the $\mathrm{H}_{\mathrm{c}}$ and $\mathrm{M}_{\mathrm{r}}$ value for the compound $\mathrm{Co}_{1.5}\left[\mathrm{Fe}(\mathrm{CN})_{6}\right] \cdot 6 \mathrm{H}_{2} \mathrm{O}\left(5 \mathrm{~K}, \mathrm{M}_{\mathrm{r}}=0.64 \mu_{\beta}, \mathrm{H}_{\mathrm{c}}=480\right.$ $\mathrm{Oe})^{8}$. Magnetic parameters like saturation magnetization $\left(\mathrm{M}_{\mathrm{s}}\right)$, coercive field (Hc), Curie constant (C), Weiss paramagnetic Curie temperature $(\theta)$, transition temperature $\left(T_{c}\right)$, are different from the those of the bimetallic cyanide-bridged compounds $\mathrm{Mn}_{1.5}\left[\mathrm{Fe}(\mathrm{CN})_{6}\right] \cdot \mathrm{xH}_{2} \mathrm{O}, \mathrm{Co}_{1.5}\left[\mathrm{Fe}(\mathrm{CN})_{6}\right] \cdot \mathrm{xH}_{2} \mathrm{O}$. Therefore,

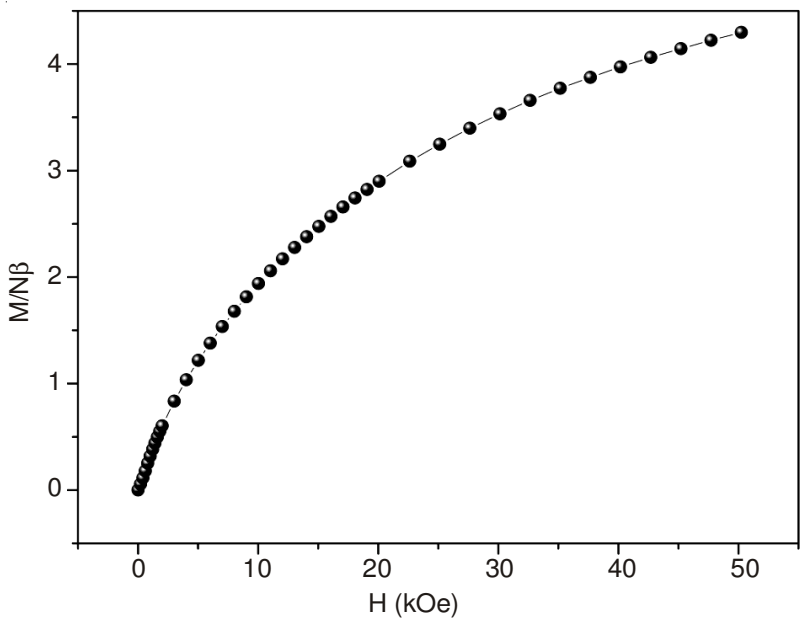

Fig. 9. Field-dependent magnetization curves

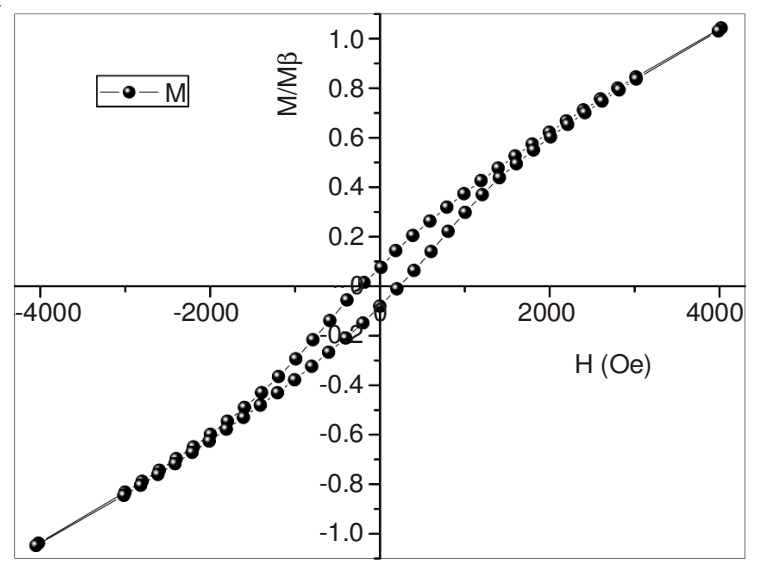

Fig. 10. Hystersis loop for the compound synthesis ideas of molecular alloy magnet can be regarded as a synthesis method to expand a new type of magnetic functional materials, which magnetic properties can be tuned and controlled by changing the composition of different transition metal cations s $^{15,17,20}$.

Measurement of Mössbauer spectrum: The room temperature Mössbauer spectroscopy of the compound have been measured (Fig. 11). The value of isomer shift (IS), quadrupole splitting (QS) and line width are listed in Table-1. Presumably the rapid motion of the magnetic iron electronic moments causes the quenching of the magnetic hyperfine structure. The observed values (Table-1) for the isomer shift, line width is $0.36(0) \mathrm{mm} / \mathrm{s}$ at $298 \mathrm{~K}$, as shown in Fig. 12. The quadrupole splitting is $0.41(9) \mathrm{mm} / \mathrm{s}$, I.S.is $-0.15(4) \mathrm{mm} / \mathrm{s}$, values which are characteristic of low-spin $\mathrm{Fe}(\mathrm{III})(\mathrm{S}=1 / 2)$ ions ${ }^{19-21}$.
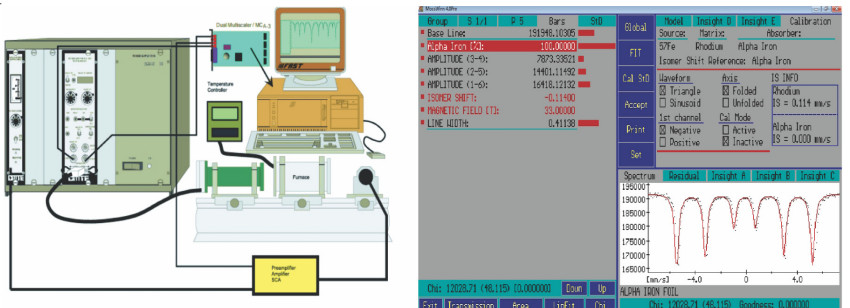

Fig. 11. Mössbauer spectrum measurement

\begin{tabular}{|c|c|}
\hline \multicolumn{2}{|c|}{$\begin{array}{l}\text { TABLE-1 } \\
\text { MÖSSBAUER PARAMETER OF THE } \\
\text { SAMPLE } \mathrm{Co}_{0.25} \mathrm{Mn}_{1.25}\left[\mathrm{Fe}(\mathrm{CN})_{6}\right] \cdot 5.9 \mathrm{H}_{2} \mathrm{O}\end{array}$} \\
\hline Temperature (K) & $300 \mathrm{~K}$ \\
\hline IS $(\mathrm{mm} / \mathrm{s})$ & $-0.15(4)$ \\
\hline QS $(\mathrm{mm} / \mathrm{s})$ & $0.41(9)$ \\
\hline Line width $(\mathrm{mm} / \mathrm{s})$ & $0.36(0)$ \\
\hline
\end{tabular}

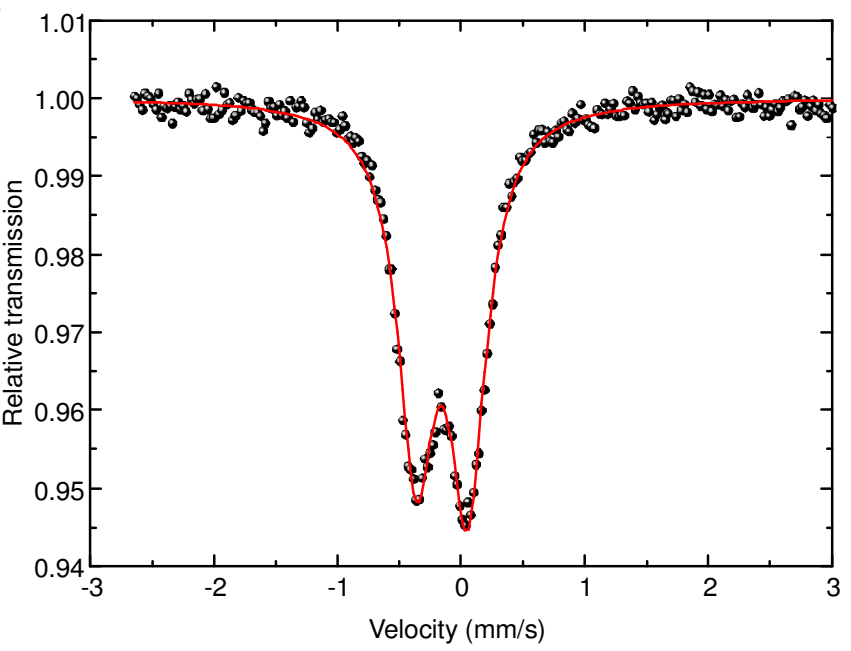

Fig. 12. Mössbauer parameters of $\mathrm{Co}_{0.25} \mathrm{Mn}_{1.25}\left[\mathrm{Fe}(\mathrm{CN})_{6}\right] \cdot 5.9 \mathrm{H}_{2} \mathrm{O}$

\section{Conclusion}

An investigation of multi-metal compound $\mathrm{Co}_{0.25} \mathrm{Mn}_{1.25}\left[\mathrm{Fe}(\mathrm{CN})_{6}\right] \cdot 5.9 \mathrm{H}_{2} \mathrm{O}$ with spin-glass behaviour and ferromagnet are reported. The compounds exhibits spontaneous magnetic ordering at low temperatures; in the temperature range $30-300 \mathrm{~K}$, the magnetic susceptibilities can be fit 
to the Curie-Weiss law with a Curie constant of $\mathrm{C}=6.59 \mathrm{~cm}^{3}$ $\mathrm{K} \mathrm{mol}^{-1}$ and Curie-Weiss temperature of $\Theta=-11.28 \mathrm{~K}$. The Mössbauer result of indicate that the isomer shift, line width is $0.36(0) \mathrm{mm} / \mathrm{s}$ at $298 \mathrm{~K}$. The quadrupole splitting is $0.41(9)$ $\mathrm{mm} / \mathrm{s}$, I.S. is $-0.15(4) \mathrm{mm} / \mathrm{s}$, values which are characteristic of low-spin $\mathrm{Fe}(\mathrm{III})(\mathrm{S}=1 / 2)$ ions.Bifurcation phenomenon of ZFC-FC DC magnetization, the maximum of magnetic susceptibility shifts toward higher temperatures with the frequencies $(32-1000 \mathrm{~Hz})$ indicate spin-glass state in the Sample $\mathrm{Co}_{0.25} \mathrm{Mn}_{1.25}\left[\mathrm{Fe}(\mathrm{CN})_{6}\right] \cdot 5.9 \mathrm{H}_{2} \mathrm{O}$, which mainly due to domain mobility growth under different cooling conditions.

\section{ACKNOWLEDGEMENTS}

This work was financially supported by the National Natural Science Foundation of China (No. 11164002, 11364004); NSF of Guangxi Province (No. 0991092).

\section{REFERENCES}

1. S. Ohkoshi, T. Iyoda, A. Fujishima and K. Hashimoto, Phys. Rev. B, 56, 11642 (1997).

2. O. Sato, T. Iyoda, A. Fujishima and K. Hashimoto, Science, 272, 704 (1996)
3. S. Ohkoshi, O. Sato, T. Iyoda, A. Fujishima and K. Hashimoto, Inorg. Chem., 36, 268 (1997).

4. O. Sato, T. Iyoda, A. Fujishima and K. Hashimoto, Science, 271, 49 (1996).

5. D. Zhang, Z. Zhao and X. Chen, Asian J. Chem., 25, 3509 (2013).

6. S. Ohkoshi, T. Hozumi and K. Hashimoto, Phys. Rev. B, 64, 132404 (2001).

7. A. Kumar and S.M. Yusuf, Physica B, 362, 278 (2005).

8. O. Sato, Y. Einaga, A. Fujishima and K. Hashimoto, Inorg. Chem., 38, 4405 (1999).

9. O. Kahn, Molecular Magnetism, VCH Publishers, New York (1993).

10. W.E. Buschmann and J.S. Miller, Inorg. Chem., 39, 2411 (2000).

11. S.M. Holmes and G.S. Girolami, J. Am. Chem. Soc., 121, 5593 (1999).

12. A. Kumar, S.M. Yusuf and L. Keller, Physica B, 385-386, 444 (2006).

13. J.S. Miller and A.J. Epstein, Angew. Chem. Int. Ed., 33, 385 (1994).

14. M. Gabay and G. Toulouse, Phys. Rev. Lett., 47, 201 (1981).

15. M. Ohba and H. Okawa, Coord. Chem. Rev., 198, 313 (2000).

16. H.Z. Kou, S. Gao and X. Jin, Synth. Inorg. Chem., 40, 6295 (2001).

17. C.A. Chugh and D. Bharti, Asian J. Chem., 24, 5879 (2012).

18. D. Sherrington and S. Kirkpatrick, Phys. Rev. Lett., 35, 1792 (1975).

19. K. Chandra, D. Raj and S.P. Puri, J. Chem. Phys., 46, 1466 (1967).

20. Q. Lin, Z. Ye, C. Lei, H. Huang, R. Wang and Y. He, Mater. Res. Innov., 17(s1), s255 (2013).

21. S. Iijima, Z. Honda, S. Koner and F. Mizutani, J. Magn. Magn. Mater, 223, 16 (2001). 